\title{
A Case of Difficult Management of Fluid-electrolyte Imbalance in Choroid Plexus Papilloma
}

\author{
Atsushi SAITO, ${ }^{1}$ Shinjitsu NiSHIMURA, ${ }^{1}$ Tomoaki FUJITA, ${ }^{1}$ Tatsuya SASAKI, ${ }^{1}$ \\ and Michiharu NISHIJIMA ${ }^{1}$
}

${ }^{1}$ Department of Neurosurgery, Aomori Prefectural Central Hospital, Aomori, Aomori

\begin{abstract}
A 22-month-old boy presented with nausea and gradual deterioration of gait disturbance. Computed tomography (CT) demonstrated an intraventricular mass lesion in the right lateral ventricle. He was referred to our department 3 weeks after onset. Acute hydrocephalus gradually proceeded 4 days after admission, and external ventricular drainage (EVD) was performed. EVD revealed cerebrospinal fluid (CSF) overproduction (800-1,500 mL/day) under constant pressure of $10 \mathrm{~cm} \mathrm{H}_{2} \mathrm{O}$ above external auditory meatus. Magnetic resonance imaging showed a multi-lobular mass in the inferior horn of the right lateral ventricle. A choroid plexus tumor was suspected. The ratio of blood urea nitrogen:creatinine (BUN:Cre) remained between 30 and 40 , and hemoglobin was between $14.0-17.0 \mathrm{mg} / \mathrm{dL}$, suggesting marked dehydration. Serum sodium varied between 117 and $140 \mathrm{mmol} / \mathrm{L}$, and serum potassium between $2.2 \mathrm{mmol} / \mathrm{L}$ and $6.9 \mathrm{mmol} / \mathrm{L}$. The amount of EVD was unstable and fluid balance management was difficult. Hypotonic fluid with sodium chloride supplement was used to adjust thefluid and electrolyte imbalance. Surgical removal of the tumor was performed6 days after EVD and tumor was grossly and totally removed. The high BUN:Cre ratio decreased to about 15 and hemoglobin recovered to $7.5-9.0 \mathrm{mg} / \mathrm{dL}$ after removal. Electrolytes returned to the normal range. Overproduction of CSF also markedly improved to $<300 \mathrm{~mL} / \mathrm{day}$. Histopathological examination diagnosed choroid plexus papilloma.

We experienced a case of choroid plexus papilloma associated with fluid-electrolyte imbalance due to over-drainage after EVD, which could not be effectively controlled before tumor removal. Cautious fluid management and emergent surgical resection might be required to managethe overproduction of CSF and fluid-electrolyteimbalance.
\end{abstract}

Key words: choroid plexus papilloma, cerebrospinal fluid, dehydration

\section{Introduction}

Hydrocephalus is a common pediatric neurosurgical problem that is usually successfully treated by ventricular shunt placement. ${ }^{11}$ Choroid plexus tumors such as choroid plexus papillomas or carcinomasare often associated with hydrocephalus due to cerebrospinal fluid (CSF) overproduction and an obstructive mass effect. ${ }^{1,2)}$ In fact, overproduction of CSF is a unique phenomenon of tumors and choroid hyperplasia. ${ }^{3)}$

We experienced a case of choroid plexus papilloma associated with fluid-electrolyte imbalance due to overdrainage of CSF after external ventricular drainage (EVD), and controlled the imbalance before tumor removal with difficulty. We report and discuss the clinical features with a literature review.

Received October 29, 2012; Accepted April 8, 2013

\section{Case Report}

A 22-month-old boy was taken to a local pediatric clinic because of nausea and lethargy. The boy's medical history was otherwise unremarkable. Vomiting, gait disturbance, and lethargy gradually increased over 2 weeks and he was referred to the Department of Pediatrics of our hospital. On physical examination, the boy was lethargic but responsive to verbal and visual stimuli. Limb muscle tone was reduced, but no definite weakness was found. Serum sodium and potassium levels were 140 and $4.1 \mathrm{mmol} / \mathrm{L}$, respectively. A computed tomography scan revealed hydrocephalus and an intraventricular tumor in the inferior horn of the right lateral ventricle. Magnetic resonance imaging (MRI) also showed a well-defined, enhanced mass with a cauliflower shape and internal papillary structures in the inferior horn of the right lateral ventricle (Fig. 1). The child was diagnosed with choroid 


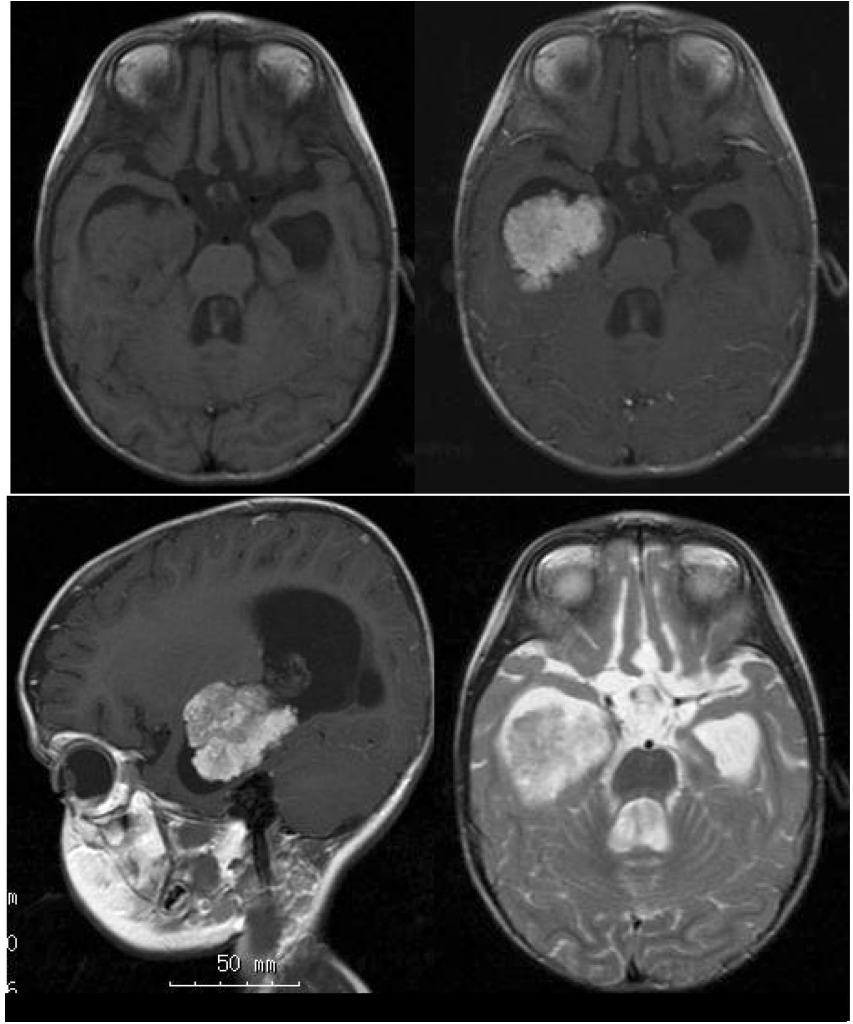

Fig. 1 Magnetic resonance imaging shows a well-defined, enhanced mass with a cauliflower shape and internal papillary structures in the inferior horn of the right lateral ventricle (Upper, left: axial $\mathrm{T}_{1}$-weighted image, right: gadolinium (Gd)enhanced image. Lower, left: sagittal Gd-enhanced image, right: axial $\mathrm{T}_{2}$-weighted image). plexus tumor and accompanying hydrocephalus. Ventriculomegaly rapidly aggravated over 4 days after admission and emergent EVD was performed to reduce intracranial pressure. Pressure of CSF drainage was set constantly at $10 \mathrm{~cm} \mathrm{H}_{2} \mathrm{O}$ above external auditory meatus to maintain appropriate intracranial pressure. Acute hydrocephalus, not compression due to the tumor mass, was suspected as the reason for consciousness disturbance. We planned to adjust intracranial pressure and systemic fluid balance first and surgical removal of the tumor was planned after adjustment of the systemic condition at that point. The amount of CSF drainage varied between $840 \mathrm{~mL} /$ day and $1,520 \mathrm{~mL} /$ day under constant pressure, serum sodium was in the range of $117-137 \mathrm{mmol} / \mathrm{L}$ and potassium was in the range of $2.2-6.9 \mathrm{mmol} / \mathrm{L}$ for 6 days after drainage (Fig. 2). Hemoglobin varied between 8.0 and 17.0, and the blood urea nitrogen:creatinine (BUN:Cre) ratio varied between 25.0 and 43.0. The supplementary fluid was hypotonic fluid containing sodium $90 \mathrm{mEq} / \mathrm{L}$ and chloride $70 \mathrm{mEq} / \mathrm{L}$ with glucose, and 600-2,400 mL per day was supplied for 6 days after drainage to correct the dehydration and electrolyte imbalance. Sodium chloride (up to $12 \mathrm{~g}$ ) was cautiously added to hypotonic fluid according to the frequent evaluation of electrolytes. With gradual normalization of the sodium-potassium imbalance and volume loss, the patient fully regained consciousness. Vomiting occurred 1 day after drainage and gradually improved and diarrhea was not observed after drainage. Oral intake recovered at the extent of $50 \%$ at the onset of hydrocephalus before tumor removal. The tumor was

$\operatorname{EVD}(\mathrm{mL} /$ day $)$

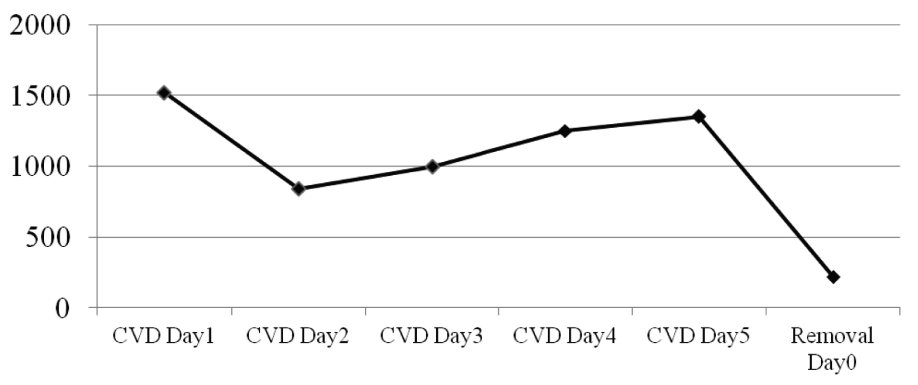

\begin{tabular}{ccccccc}
\hline Serum Na (mmol/L) & 140 & 124 & 117 & 128 & 117 & 137 \\
Serum K (mmol/L) & 4.1 & 5 & 6.9 & 2.2 & 4.5 & 3.8 \\
Serum Cl (mmol/L) & 104 & 86 & 81 & 103 & 87 & 105 \\
In (mL/day) & 2700 & 2500 & 2500 & 2700 & 3200 & 1700 \\
WB (mL/day) & 750 & 620 & -220 & -130 & 230 & 250 \\
Na in fluid (mEq) & 206 & 188 & 194 & 210 & 246 & 130 \\
K in fluid (mEq) & 72 & 62 & 68 & 68 & 88 & 42 \\
BW(kg) & 10.1 & 9.8 & 9.6 & 9.4 & 9.5 & 9.6 \\
Vomiting & ++ & + & + & - & - & - \\
Diarrhea & - & - & - & - & - & -
\end{tabular}

Fig. 2 Time-dependent change of the amount of external ventricular drainage (EVD) and electrolytes of serum sodium, potassium and chloride after cerebral ventricular drainage (CVD). Upper line graph demonstrates over-drainage of cerebrospinal fluid and instability of the amount after CVD. Over-drainage improved after tumor removal. Lower panels showed marked change of serum sodium ( $\mathrm{Na}$ ), potassium (K) and chloride ( $\mathrm{Cl}$ ), and changes of water balance (WB), body weight (BW). Vomiting + shows grade of slight and ++ shows moderately severe. The unstable electrolytes were also corrected after tumor removal. 
removed 6 days after drainage. During the operation, a reddish hypervascular tumor with a gross papillary structure was observed and the tumor was completely removed. Pathological examination revealed it to be a choroid plexus papilloma (Fig. 3). Postoperative MRI demonstrated total removal of the lesion (Fig. 4). The amount of CSF drainage gradually reduced; however, the daily amount of CSF did not fall below $50 \mathrm{~mL}$ and occlusion of CSF drainage slowly caused ventricle enlargement. Ventriculo-peritoneal (VP) shunt was performed 3 weeks after removal. Shunt pressure was controlled under $10 \mathrm{~cm} \mathrm{H}_{2} \mathrm{O}$ and hydrocephalus did not recur after VP shunt placement. The patient was discharged without neurological deficits 2 months after VP shunt placement. No tumor recurrence has been observed for 4 years after removal.

\section{Discussion}

Choroid plexus papillomas are rare, accounting for less than $1 \%$ of all intracranial tumors in adults; ${ }^{4)}$ however, they are relatively more common duringchildhood and constitute 1.5 to $4 \%$ of intracranial tumors. ${ }^{1)}$ They are most often located in the lateral ventricle, followed by the fourth and third ventricles and, rarely, in the cerebellopontine angle.$^{5)}$ Overproduction of CSF causing hydrocephalus is a well-known phenomenon of choroid plexus papilloma, although obstruction of CSF pathways may also cause non-communicating hydrocephalus in some patients. ${ }^{6}$ Eisenberg et al. reported the rate of CSF formation to be $1.4 \mathrm{~mL} / \mathrm{min}$, or more than $2 \mathrm{~L} /$ day, in a 5 -month-old baby with choroid plexus papilloma and hydrocephalus using a ventricular perfusion technique. ${ }^{7)}$ Considering the role of the normal choroid plexus in CSF production and the size of the choroid plexus papilloma, CSF

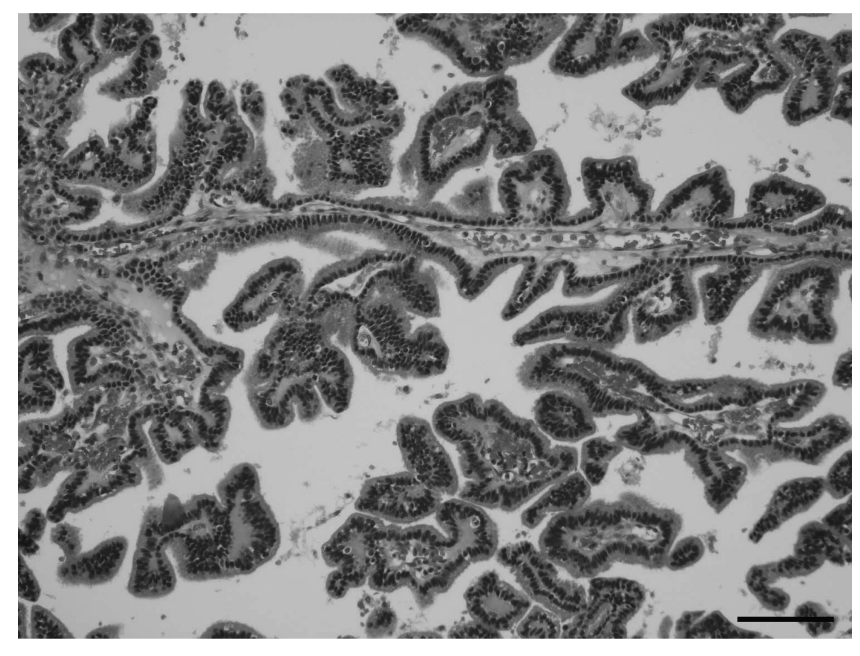

Fig. 3 Microscopic photograph of hematoxylin-eosin staining of a pathological specimen shows tumor cells arranged in papillary structures $(\times 100$. Bar, $200 \mu \mathrm{m})$. production rates many times greater than normal are not uncommon. ${ }^{2,3)}$ For instance, CSF production of $5 \mathrm{~L} /$ day has been reported for a patient with bilateral choroid plexus papillomas. ${ }^{1)}$

In our case, it was difficult to manage the electrolyte imbalance and dehydration. Tumor removal was performed after correction of fluid balance. Infants are vulnerable to water and electrolyte imbalances. ${ }^{3)}$ Even a day of starvation or repeated vomiting can cause severe water and electrolyte imbalance in this population. ${ }^{3)} \mathrm{CSF}$ is an isotonic fluid with an approximate sodium concentration of $138 \mathrm{mmol} / \mathrm{L}$, but the potassium content of CSF is $2.8 \mathrm{mmol} / \mathrm{L}$, which is less than that of extracellular fluid $(4.5 \mathrm{mmol} / \mathrm{L}){ }^{8)}$ To date, hypotonic saline with a physiologic potassium supplement has been widely used as maintenance fluid in pediatrics; ${ }^{3,9)}$ however, many recent studies have criticized this tradition of prescribing hypotonic saline for sick children because of the risk of developing iatrogenic hyponatremia, which is often fatal. ${ }^{3,10,11)}$ In the present case, our first strategy of tumor removal after correction of the fluid-electrolyte balance might be questionable as a result. The tumor caused unstable overproduction of CSF and drainage of CSF was required to prevent hydrocephalus. Another questionable point was external CSF drainage.
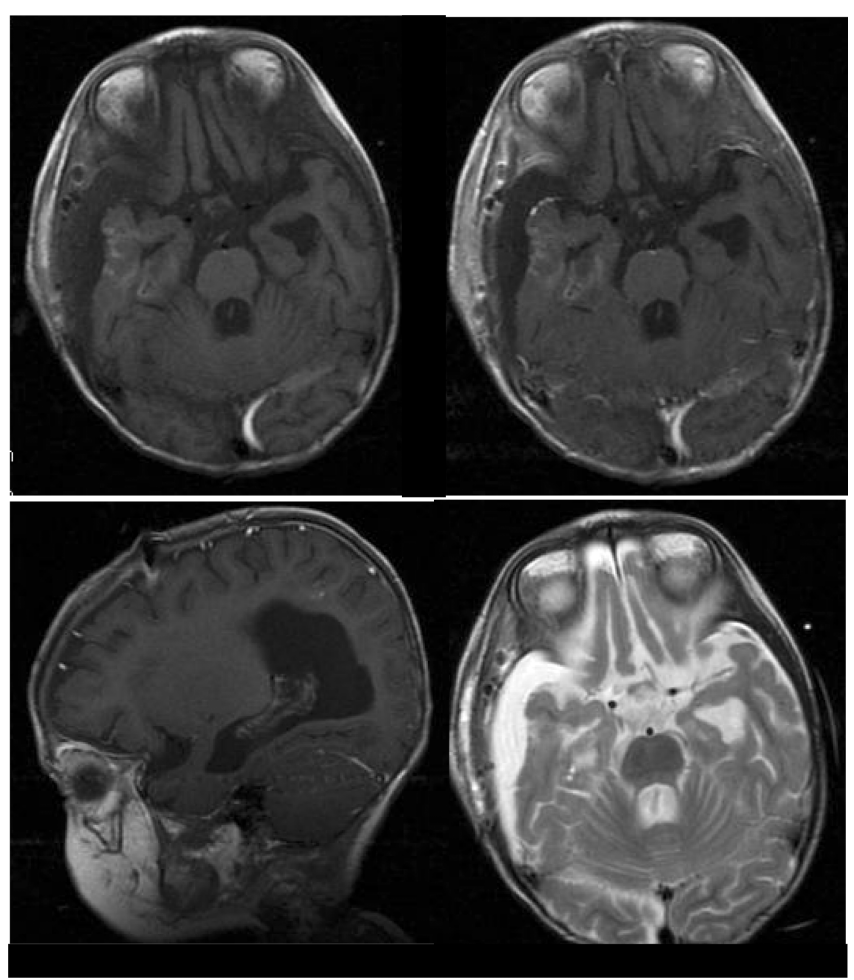

Fig. 4 Postoperative magnetic resonance imaging shows no remnant enhancing mass in the inferior horn of the right lateral ventricle (Upper, left: axial $\mathrm{T}_{1}$-weighted image, right: gadolinium (Gd)-enhanced image. Lower, left: sagittal Gd-enhanced image, right: axial $\mathrm{T}_{2}$-weighted image). 
We chose emergent ventricular drainage to control acute hydrocephalus, which might have worsened the electrolyte balance. Emergent ventriculo-peritoneal (VP) shunt might be useful because of isotonic fluid return under slight CSF production and normal absorption via the peritoneum. Nimjee et al. reported a case of choroid plexus papilloma associated with CSF overproduction. ${ }^{1)}$ They discussed whether 500-600 mL/day of CSF production might not enable peritoneal resorption and selected a ventriculo-atrial shunt. ${ }^{1)}$ Finally, single-stage bilateral choroidectomy was effective incontrolling hydrocephalus. ${ }^{1)}$ Fujimura et al. also reported a case of choroid plexus papilloma associated with overproduction of CSF. ${ }^{2)}$ Their patient presented with intractable ascites after VP shunt placement and choroid plexus papilloma was diagnosed during examination for shunt malfunction. VA shunt replacement and external drainage were not effective and tumor removal relieved CSF overproduction.

Osmolarity of supplementary fluid is unknown for unstable CSF over-drainage and no report has focused on electrolyte imbalance in choroid plexus papilloma to our knowledge. We chose hypotonic fluid to correct dehydration, and supplementary administration of sodium chloride was controlled according to the frequent evaluation of laboratory data. Instability of the amount of EVD made it difficult to correct the fluid-electrolyte imbalance, which returned to the normal range after emergent tumor removal. Hyperkalemia caused by dehydration and sudden elevation of serum potassium caused by fluid supplementation should be considered for electrolyte imbalance due to CSF over-drainage. However, the sodium-potassium imbalance was unstable and was not as easy to control as urinary excretion or simple dehydration because of the unstable amount of CSF drainage and difficulty in precise evaluation of the electrolytes in the drained CSF. We could not completely examine and follow the laboratory data, and repeated evaluation of electrolytes in urine and CSF as well as that of serum electrolytes should be important. If we could accurately examine electrolyte balance in CSF as well as serum and urine, better management might be possible; however, emergent tumor removal might be effective in managing not only hydrocephalus but also systemic fluid balance.

In our case, a VP shunt was placed even after tumor removal. The tumor was totally removed and has not recurred during the 4 years since removal. Hydrocephalus after tumor removal was not aggressive and much less external CSF drainage was required than that for preoperative hydrocephalus. There is a possibility that CSF drainage might require external drainage even during short-term drainage. Fujimura et al. also reported the requirement of VP shunt placement even after tumor removal. ${ }^{2)}$ No aggravation of hydrocephalus was observed after shunt placement with constant shunt pressure.

We could not find a definitive strategy for controlling hydrocephalus due to choroid plexus papilloma in our search of the literature. Return of CSF overproduction by VP or VA shunt might not be a better treatment method from theview of CSF absorption. External drainage of CSF overproduction might be effective in controlling only ventriculomegaly; however, as shown in our present case, it might aggravate the fluid-electrolyte imbalance and make the management more difficult. Previous literature have discussed appropriate control of CSF overproduction associated with choroid plexus papilloma. They have reported that it was not easy and might be controversial. We suggest that shunt placement might be a candidate for mild CSF overproduction as preoperative management strategy for CSF overproduction in case emergenttumor removal is not permitted. External CSF drainage might be the second choice and might be selected for severe CSF overproduction or for cases where shunt placement was ineffective and as a short-term option before tumor removal, because the setting of drainage itself is controversial and is dependent on pressure or amount. Longterm over-drainage of CSF might make fluid-electrolyte imbalance worse.

\section{Conclusion}

We experienced a case of choroid plexus papilloma associated with fluid-electrolyte imbalance and could not effectively control the imbalance before tumor removal. Over-drainage of CSF after EVD and insufficient fluid balance control might cause severe fluid-electrolyte imbalance. Cautious fluid-electrolyte management before surgery and emergent removal of the tumor might be required tomanage hydrocephalus and fluid-electrolyte imbalance.

\section{Conflicts of Interest Disclosure}

The authors report no conflicts of interest concerning the case report used in this study or the findings specified in this paper.

\section{References}

1) Nimjee SM, Powers CJ, McLendon RE, Grant GA, Fuchs HE: Single-stage bilateral choroid plexectomy for choroid plexus papilloma in a patient presenting with high cerebrospinal fluid output. J Neurosurg Pediatr 5: 342-345, 2010

2) Fujimura M, Onuma T, Kameyama M, Motohashi O, Kon H, Yamamoto K, Ishii K, Tominaga T: Hydrocephalus due to cerebrospinal fluid overproduction by bilateral choroid plexus papillomas. Childs NervSyst 20: 485-488, 2004

3) Phi JH, Shin CH, Wang KC, Park SH, Kim SK: Catastrophic electrolyte imbalance caused by excessive production and overdrainage of cerebrospinal fluid in an infant with choroid plexus papilloma. Childs Nerv Syst 27: 1153-1156, 2011

4) Erman T, Göçer AI, Erdoğan S, Tuna M, Ildan F, Zorludemir 
S: Choroid plexus papilloma of bilateral lateral ventricle. Acta Neurochir (Wien) 145: 139-143; discussion 143, 2003

5) Horská A, Ulug AM, Melhem ER, Filippi CG, Burger PC, Edgar MA, Souweidane MM, Carson BS, Barker PB: Proton magnetic resonance spectroscopy of choroid plexus tumors in children. J Magn Reson Imaging 14: 78-82, 2001

6) Sahar A, Feinsod M, Beller AJ: Choroid plexus papilloma: hydrocephalus and cerebrospinal fluid dynamics. Surg Neurol 13: 476-478, 1980

7) Eisenberg HM, McComb JG, Lorenzo AV: Cerebrospinal fluid overproduction and hydrocephalus associated with choroid plexus papilloma. J Neurosurg 40: 381-385, 1974

8) Robertson DS: The physical chemistry of brain and neural cell membranes: an overview. Neurochem Res 35: 681-687, 2010

9) Davies P, Hall T, Ali T, Lakhoo K: Intravenous postoperative fluid prescriptions for children: a survey of practice. $B M C$ Surg 8: 10, 2008

10) Montañana PA, Modesto i Alapont V, Ocón AP, López PO, López Prats JL, Toledo Parreño JD: The use of isotonic fluid as maintenance therapy prevents iatrogenic hyponatremia in pediatrics: a randomized, controlled open study. Pediatr Crit Care Med 9: 589-597, 2008

11) Yung M, Keeley S: Randomised controlled trial of intravenous maintenance fluids. J Pediatr Child Health 45: 9-14, 2009

Address reprint requests to: Atsushi Saito, MD, $\mathrm{PhD}$, Department of Neurosurgery, Aomori Prefectural Central Hospital, 2-1-1 Higashitsukurimichi, Aomori, Aomori 030-0910, Japan. e-mail: satsushi2002@yahoo.co.jp 